\title{
BMJ Open Protocol for a randomised controlled implementation trial of point-of-care viral load testing and task shifting: the Simplifying HIV TREAtment and Monitoring (STREAM) study
}

Jienchi Dorward, ${ }^{1}$ Nigel Garrett, ${ }^{1,2}$ Justice Quame-Amaglo, ${ }^{3}$ Natasha Samsunder, ${ }^{1}$ Hope Ngobese, ${ }^{4}$ Noluthando Ngomane, ${ }^{4}$ Pravikrishnen Moodley, ${ }^{5}$ Koleka Mlisana, ${ }^{6,7}$ Torin Schaafsma, ${ }^{3}$ Deborah Donnell, ${ }^{3}$ Ruanne Barnabas, ${ }^{3,8,9}$ Kogieleum Naidoo, ${ }^{1,10}$ Salim Abdool Karim, ${ }^{1,10,11}$ Connie Celum, ${ }^{3,8}$ Paul K Drain ${ }^{3,8,9}$

To cite: Dorward J, Garrett N, Quame-Amaglo J, et al. Protocol for a randomised controlled implementation trial of point-of-care viral load testing and task shifting: the Simplifying HIV TREAtment and Monitoring (STREAM) study. BMJ Open 2017;7:e017507. doi:10.1136/ bmjopen-2017-017507

- Prepublication history and additional material for this paper are available online. To view please visit the journal (http:// dx.doi.org/).

Received 27 April 2017 Revised 28 August 2017 Accepted 29 August 2017

CrossMark

For numbered affiliations see end of article.

Correspondence to

Dr Paul K Drain;

pkdrain@uw.edu

\section{ABSTRACT}

Introduction Achieving the Joint United Nations Programme on HIV and AIDS 90-90-90 targets requires models of HIV care that expand antiretroviral therapy (ART) coverage without overburdening health systems. Point-of-care (POC) viral load (VL) testing has the potential to efficiently monitor ART treatment, while enrolled nurses may be able to provide safe and cost-effective chronic care for stable patients with HIV. This study aims to demonstrate whether POC VL testing combined with task shifting to enrolled nurses is non-inferior and costeffective compared with laboratory-based VL monitoring and standard HIV care.

Methods and analysis The STREAM (Simplifying HIV TREAtment and Monitoring) study is an open-label, noninferiority, randomised controlled implementation trial. HIV-positive adults, clinically stable at 6 months after ART initiation, will be recruited in a large urban clinic in South Africa. Approximately 396 participants will be randomised $1: 1$ to receive POC HIV VL monitoring and potential task shifting to enrolled nurses, versus laboratory VL monitoring and standard South African HIV care. Initial clinic follow-up will be 2-monthly in both arms, with VL testing at enrolment, 6 months and 12 months. At 6 months (1 year after ART initiation), stable participants in both arms will qualify for a differentiated care model involving decentralised ART pickup at community-based pharmacies. The primary outcome is retention in care and virological suppression at 12 months from enrolment. Secondary outcomes include time to appropriate entry into the decentralised ART delivery programme, costs per virologically suppressed patient and cost-effectiveness of the intervention compared with standard care. Findings will inform the scale up of VL testing and differentiated care in HIV-endemic resource-limited settings.

Ethics and dissemination Ethical approval has been granted by the University of KwaZulu-Natal Biomedical Research Ethics Committee (BFC296/16) and University of Washington Institutional Review Board (STUDY00001466). Results will be presented at international conferences and published in academic peer-reviewed journals.

\section{Strengths and limitations of this study}

- This is the first randomised controlled implementation trial of point-of-care HIV viral load testing and task shifting to enrolled nurses that we are aware of.

- Point-of-care testing allows viral loads to be taken and acted on in one clinic visit, while enrolled nurses may be able to provide safe and cost-effective HIV care for stable patients.

- This strategy could lessen workloads for HIV clinics in low-income and middle-income countries, thereby increasing capacity for universal antiretroviral therapy coverage and viral load monitoring in line with Joint United Nations Programme on HIV and AIDS 90-90-90 targets.

- This is a single-site study, so results may not be generalisable to other settings.

Trial registration NCT03066128; Pre-results.

\section{INTRODUCTION}

Strong evidence of the beneficial effects of early antiretroviral therapy (ART) ${ }^{1-3}$ has led to WHO recommending ART for all people living with HIV (PLHIV). ${ }^{4}$ This aligns with the Joint United Nations Programme on HIV and AIDS (UNAIDS) 90-90-90 targets (90\% of all PLHIV to know their status, $90 \%$ of these to be on ART and $90 \%$ of these to be virologically suppressed) to be achieved by the year 2020..$^{5}$ In 2016, 18.2 million PLHIV were receiving ART globally, but another 18.5 million still required treatment. ${ }^{6}$ Many health systems in low-income and middle-income countries are operating at capacity and will struggle to provide care for more 
HIV-positive patients without compromising clinical outcomes. ${ }^{7}$ Rapid scale up of ART coverage may lead to several operational issues, including longer waiting times, ART stock-outs and human resource shortages, which would jeopardise achieving the 90-90-90 targets. ${ }^{8}$ Furthermore, since current models of HIV care were designed in the era of higher HIV-related morbidity and less tolerable ART regimens, many clinics may not be providing effective, patient-centred, chronic care for healthy PLHIV. ${ }^{9} 10$

In order to meet these challenges, newer models of chronic HIV care have been developed. These 'differentiated care models ${ }^{10}{ }^{11}$ include healthcare facility and community-based strategies involving elements of task shifting, ${ }^{12}$ less frequent clinic visits ${ }^{13}$ and/or decentralised ART distribution through community pharmacies or peer support groups. ${ }^{14-17}$ Triaging stable HIV-positive patients in differentiated care models requires quantitative viral load (VL) testing, which the WHO recommends as the most effective method of monitoring the response to ART. ${ }^{4}$ Patients with suppressed VLs may be able to receive less frequent follow-up visits, allowing resources to be redirected towards those with virological failure or other ongoing health needs.

Despite requiring sophisticated laboratory services, models suggest VL monitoring is cost-effective in Southern Africa when compared with CD4 count monitoring alone or monitoring clinical signs and symptoms. ${ }^{18}$ VL monitoring should be performed at 6 and 12 months after ART initiation and then annually according to WHO guidelines. ${ }^{4}$ Laboratory capacity to provide VL testing varies across the region, with $75 \%$ of patients on ART in South Africa having at least one VL performed, but only $10 \%$ in Uganda and $38 \%$ in Kenya. ${ }^{19}$ Rapid scale up is required to provide the 10 million annual VL tests that will be needed in Africa by the year 2020. ${ }^{2021}$ There is growing interest in point-of-care (POC) VL assays, ${ }^{22-24}$ which could alleviate the burden on central laboratory services and improve clinical management by providing test results while the patient is still at the clinic, thereby removing the need for specimen transport and follow-up visits. Providing immediate VL results may facilitate timely adherence interventions and, where necessary, ART regimen changes, thereby improving viral suppression. Modelling studies suggest that POC VL testing may be cost-effective when compared with laboratory VL testing, by improving retention and facilitating differentiated care. ${ }^{2526}$

Several POC VL assays are already on the market or in development. ${ }^{27}{ }^{28}$ Qualitative assays target early infant diagnosis and acute HIV infection, while quantitative assays are better suited for monitoring ART. The Cepheid Xpert HIV-1 VL is a fully automated, nucleic acid amplification assay based on the GeneXpert platform (Cepheid, Sunnyvale, California, USA). We have validated this assay in our South African clinic, ${ }^{29}$ and subsequent studies in other Southern African settings have produced similar results. ${ }^{30-32}$ However, the clinical effectiveness of the Xpert HIV-1 VL, as well as its potential role in differentiated care models, has yet to be demonstrated.
In South Africa and other African countries, enrolled (or associate) nurses are less highly trained than professional nurses and are cheaper to train and employ. ${ }^{33} 34$ Despite being able to perform aspects of chronic HIV care, such as basic clinical assessments, counsel patients, perform venipuncture and operate POC tests, enrolled nurses currently do not have a prominent role in clinical management of ART. We hypothesise that enrolled nurses can provide safe and cost-effective care to stable HIV-positive patients, while POC VL testing can improve management of virological failure and efficiently triage patients into differentiated care.

\section{Objectives}

The goal of the STREAM (Simplifying HIV TREAtment and Monitoring) study is to demonstrate whether POC HIV VL testing combined with task shifting to enrolled nurses is non-inferior and cost-effective compared with laboratory-based VL monitoring and standard HIV care in South Africa.

\section{METHODS AND ANALYSIS \\ Trial design}

The STREAM study is a single-site, open-label, two-arm, non-inferiority, randomised controlled implementation trial. HIV-positive adults who are clinically stable at 6 months after ART initiation will be randomised 1:1 to receive POC VL monitoring and potential task shifting to enrolled nurses, versus laboratory-based VL monitoring and standard South African HIV care. ${ }^{35}$ The primary outcome is retention in care and virological suppression at 12 months from enrolment (see figure 1 for CONSORT diagram).

\section{Study setting}

The study will take place at the Centre for the AIDS Programme of Research in South Africa (CAPRISA) eThekwini Clinical Research Site and the adjacent Prince Cyril Zulu Communicable Disease Clinic (PCZ CDC), a large public healthcare clinic in central Durban, KwaZulu-Natal, South Africa. KwaZulu-Natal is a hyper-endemic setting with an HIV prevalence of $28 \%$ among adults aged $15-49 .{ }^{36}$ PCZ CDC cares for over 10000 HIV-positive patients and also provides tuberculosis (TB) treatment, family planning, sexual health and primary care services. CAPRISA eThekwini Clinical Research Site conducts HIV treatment and prevention trials. The two clinics are situated near the main transport hub for public commuters in central Durban and serve a diverse, mobile population. PCZ CDC provides treatment in accordance with South African guidelines that require all PLHIV to be offered ART, irrespective of CD4 count, since September 2016. ${ }^{37}$ Routine care of HIV, sexual and reproductive health, minor illnesses and stable non-communicable diseases is provided by professional nurses, with referral to a physician for management of virological failure, adverse events, opportunistic infections and poorly controlled 


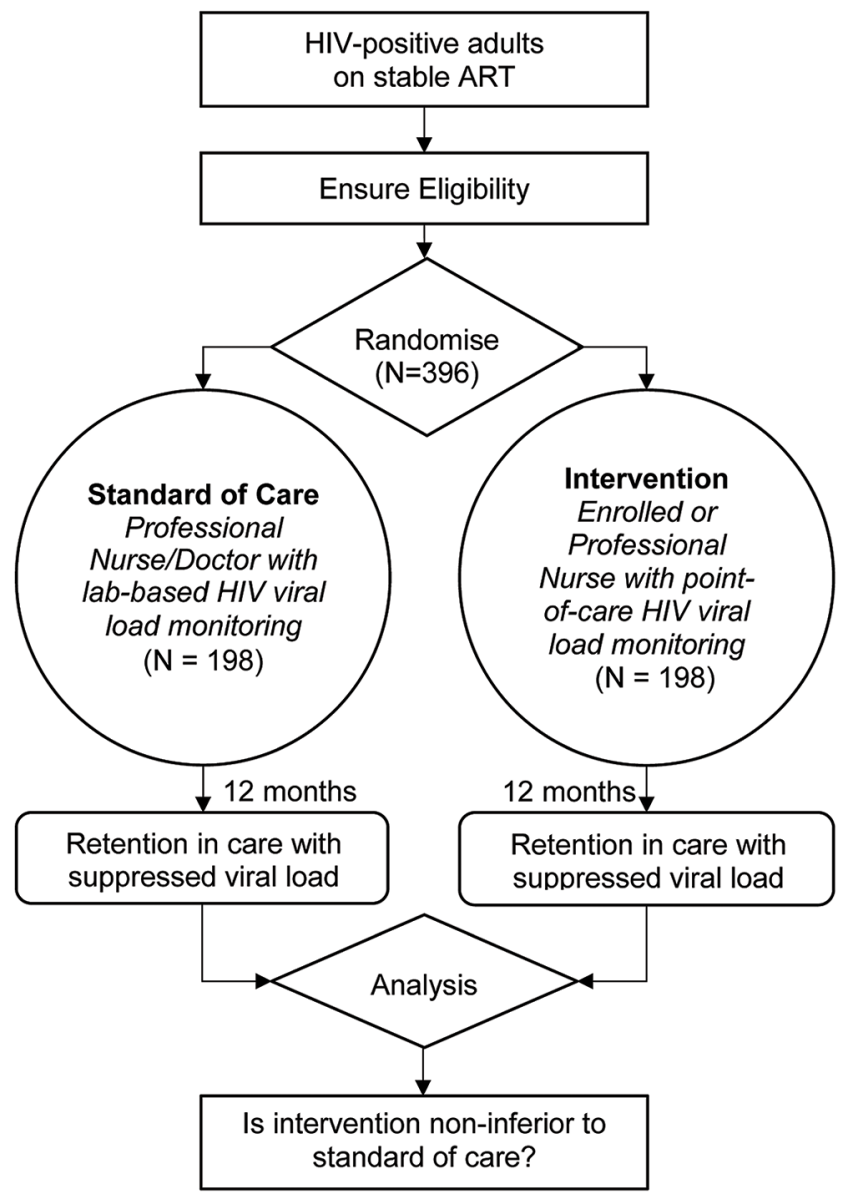

Figure 1 CONSORT diagram of the STREAM study. ART, antiretroviral therapy; STREAM, Simplifying HIV TREAtment and Monitoring.

non-communicable diseases. In line with South African national policy, PCZ CDC operates a decentralised differentiated care model, in which stable patients are referred to the Central Chronic Medicine Dispensing and Distribution Programme (CCMDD). ${ }^{37}$ This involves 2-monthly ART collection at community pharmacies and 6-monthly clinic visits with a professional nurse or physician (figure 2).
Eligibility criteria

HIV-positive adults, 18 years or older, who receive ART from PCZ CDC will be recruited when presenting for their first routine VL test, which is 6 months after ART initiation. Stable patients who plan to receive care at PCZ CDC for the following year and are willing and able to provide written informed consent will be eligible for enrolment. Patients who have already had their 6-month VL performed, are pregnant, have TB or require active care by a physician in accordance with South African guidelines $^{39}$ (see online supplementary table 1) will be excluded from the study. These eligibility criteria are meant to select stable patients who are best suited to receive differentiated HIV care.

\section{Randomisation}

The study statistician has generated the allocation sequence using random numbers generated in SAS V.9.4 (SAS Institute). Sequentially numbered, sealed, opaque envelopes containing intervention allocation and participant identification number have been given to the study clinical team, to be opened once a participant has been deemed eligible and is ready to be enrolled into the study. To ensure that the allocation sequence is followed, study nurses performing enrolment procedures will not open randomisation envelopes; this will be performed by the study coordinator, with subsequent checks to verify correct allocation.

\section{Study procedures}

At enrolment, participants will be asked to provide information on their demographic, social and medical history, including age, education level, income, employment, relationship status, HIV disclosure and travel to clinic. Locally validated instruments to measure risk factors for virological failure will be used including the WHO Alcohol Use Disorder Identification Tool (AUDIT-C), ${ }^{40}$ WHO Violence Against Women Instrument (female participants only) ${ }^{42} 43$ and the Patient Health Questionnaire 2 screen for depression. ${ }^{445}$

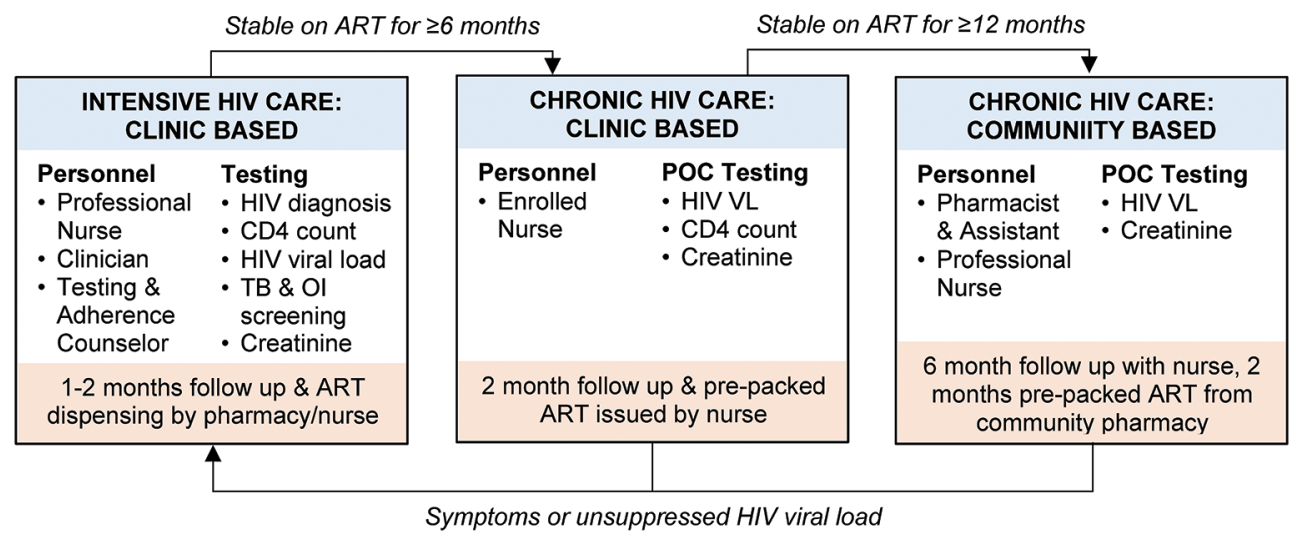

Figure 2 Conceptual model of differentiated care in the STREAM study. ART, antiretroviral therapy; OI, opportunistic infection; POC, point of care; STREAM, Simplifying HIV TREAtment and Monitoring; TB, tuberculosis; VL, viral load. 
Table 1 Schedule of evaluation in the STREAM study

\begin{tabular}{|c|c|c|c|c|c|c|c|c|}
\hline & & \multicolumn{7}{|c|}{ Study period } \\
\hline & & Enrolment & Foll & & & & & Exit \\
\hline \multicolumn{2}{|l|}{ Months in study } & 0 & 2 & 4 & 6 & 8 & 10 & 12 \\
\hline \multicolumn{2}{|l|}{ Months on ART } & 6 & 8 & 10 & 12 & 14 & 16 & 18 \\
\hline \multicolumn{2}{|l|}{ Eligibility screen } & $x$ & & & & & & \\
\hline \multicolumn{2}{|l|}{ Informed consent } & $x$ & & & & & & \\
\hline \multicolumn{2}{|l|}{ Randomisation } & $x$ & & & & & & \\
\hline \multirow[t]{2}{*}{ Intervention arm } & POC bloods & VL, Cr & & & $\begin{array}{l}\text { VL, Cr, } \\
\text { CD4 }\end{array}$ & & & $\mathrm{VL}, \mathrm{Cr}$ \\
\hline & Care provider & PN & EN & EN & PN & $\mathrm{EN}^{*}$ & $\mathrm{EN}^{*}$ & PN \\
\hline \multirow[t]{2}{*}{$\begin{array}{l}\mathrm{SOC} \\
\text { arm }\end{array}$} & Lab bloods & VL, Cr & & & $\begin{array}{l}\text { VL, Cr, } \\
\text { CD4 }\end{array}$ & & & VL, Cr \\
\hline & Care provider & PN & PN & PN & PN & $\mathrm{PN}^{*}$ & $\mathrm{PN}^{*}$ & PN \\
\hline \multicolumn{2}{|c|}{ Demographics and social questionnaire } & $\mathrm{x}$ & & & & & & \\
\hline \multicolumn{2}{|c|}{$\begin{array}{l}\text { Retention in care and virological } \\
\text { suppressiont }\end{array}$} & & & & & & & $x$ \\
\hline \multicolumn{2}{|c|}{$\begin{array}{l}\text { Symptom screen, vital signs, adherence } \\
\text { assessment }\end{array}$} & $\mathrm{x}$ & $\mathrm{x}$ & $x$ & $\mathrm{x}$ & $X^{*}$ & $X^{*}$ & $\mathrm{x}$ \\
\hline \multicolumn{2}{|c|}{ Laboratory full blood count, CD4, urinalysis } & $x$ & & & & & & $x$ \\
\hline \multicolumn{2}{|l|}{ Stored blood } & $\mathrm{x}$ & & & $\mathrm{x}$ & & & $\mathrm{x}$ \\
\hline
\end{tabular}

${ }^{*}$ No clinic visits at 8 and 10 months, if a participant was successfully referred into the community pharmacy pickup CCMDD programme at 6 months.

†Virological suppression measured using Roche Taqman V.2.0 in both arms.

ART, antiretroviral therapy; Cr, creatinine; EN, enrolled nurse; PN, professional nurse; POC, point of care; SOC, standard of care; STREAM, Simplifying HIV TREAtment and Monitoring; VL, viral load.

Participants randomised to the standard of care arm will have laboratory-based VL, creatinine and CD4 count monitoring and clinical visits performed at PCZ CDC as scheduled in the South African Department of Health HIV guidelines, with laboratory results provided at their next clinic attendance. ${ }^{35}$ Laboratory VL and creatinine will be taken at enrolment, which is 6 months after ART initiation, with subsequent 2-monthly clinical visits by a professional nurse or physician (table 1). After 6 months, laboratory VL, creatinine and CD4 will be taken.

Participants randomised to the intervention arm will have the same schedule of routine blood monitoring performed, but using POC assays: Xpert HIV-1 VL, serum creatinine (Statsensor Xpress-i, Nova Biomedical, Waltham, Massachusetts, USA) and CD4 count (Pima, Alere, Waltham, Massachusetts, USA). The Statsensor Xpress- $\mathrm{i}^{46-48}$ and Pima ${ }^{49} 50$ have been validated in multiple settings and will also be validated locally as part of trial preparations. Use of these assays, in addition to Xpert HIV-1 VL, will allow patients to have all routine bloods drawn and results provided in the same visit. POC VL and creatinine will be performed at enrolment, with results reviewed at that visit by a professional nurse. Patients who are virologically suppressed and with no comorbidities will be referred to see an enrolled nurse at subsequent visits, rather than a professional nurse, which constitutes the task shifting component of the intervention. The enrolled nurse will receive training in basic HIV/TB management, ART and side effects, adherence counselling and appropriate referral systems. At 2-monthly visits, he/she will perform vital signs, screen for TB, assess adherence and issue predispensed ART packages. If the enrolled nurse detects symptoms or abnormal vital signs, the patient will be referred to a professional nurse for review at the same visit. After 6 months in the study, which is 1 year after ART initiation, a professional nurse will assess POC VL, creatinine and CD4 count.

\section{Decentralised ART delivery (CCMDD)}

Participants in both arms who remain virologically suppressed, have CD4 count $>200$ cells $/ \mathrm{mm}^{3}$ and are clinically stable at 1 year after ART initiation will be referred into CCMDD by a professional nurse. Here they will receive 2-monthly community-based pharmacy pickup and clinic review after 6 months. Patients not eligible for CCMDD will continue with 2-monthly clinic visits with a professional nurse.

\section{Virological failure}

Management of patients with a VL of $>1000$ copies $/ \mathrm{mL}$ will follow South African guidelines in both arms, with intensive adherence counselling and repeat VL testing after 2 months (POC Xpert HIV-1 VL in the intervention $\mathrm{arm})$. If the repeat VL remains $>1000$ copies $/ \mathrm{mL}$ and 
adherence issues have been addressed, the patient will be switched to standard second-line ART by a physician.

\section{Additional laboratory investigations}

Participants in both arms will have laboratory CD4 count (FACSCalibur, BD Biosciences, San Jose, California, USA), full blood count and urinalysis performed at enrolment and at study exit. Plasma will be drawn for storage at enrolment, month 6 and study exit, to allow retrospective HIV drug resistance testing at study completion.

\section{Primary outcome}

The primary outcome is a composite measure of retention in care and virological suppression at 12 months after study enrolment (18 months on ART). Retention in care is defined as collecting ART at the 12-month study visit, while virological suppression is defined as a $\mathrm{VL}<200$ copies $/ \mathrm{mL}$ using the Roche Taqman V.2.0 (Roche Diagnostics, Basel, Switzerland). This assay will be used in both arms at 12 months to allow direct comparison.

\section{Secondary outcomes}

Secondary outcomes comparing standard of care and intervention arms are the following:

- Proportion with HIV VL $<200$ copies/mL at 12 months after enrolment.

- Proportion retained in care at 12 months after enrolment.

- Proportion appropriately retained on same ART regimen throughout study duration.

- Mean change in CD4 count from enrolment to 12-month study exit visit.

- Costs incurred per patient and per patient virologically suppressed and retained in care.

- Time to detection of virological failure, subsequent intensive adherence counselling and initiation of second-line regimen.

- Proportion of lost to follow-up or deceased (established using hospital records, death certificate and family verbal report).

- Proportion of patients entered appropriately into CCMDD and time to appropriate entry into CCMDD.

- Mean number of clinical visits per patient.

\section{Sample size}

The sample size and power calculation are based on determining non-inferiority of the intervention arm (table 2). Based on the clinic's historical performance, $80 \%$ of adults in the standard of care arm will be virologically suppressed and retained at 12 months. With a non-inferiority margin of $-10 \%$, which would be acceptable if the intervention is less costly, a trial of 198 participants per arm will have $80 \%$ power with the lower limit of a one-sided $95 \%$ CI to declare non-inferiority.

\section{Recruitment}

Recruitment of approximately 396 participants is planned over 6 months, requiring a minimum of 3 participants per working day. Study information in the form of leaflets and short presentations will be presented to patients in the PCZ CDC waiting area. Clinic staff will be trained regarding study procedures, eligibility criteria and how to refer eligible patients for screening. If necessary, study staff will also actively recruit interested patients in the clinic waiting area.

\section{Retention activities}

Participants in both arms who are more than 2 weeks late for a scheduled clinic or community pharmacy visit will receive one attempt at telephonic follow-up, as per South African guidelines. ${ }^{35}$ Community pharmacies will inform PCZ CDC of participants in CCMDD who are more than 2 weeks late for ART collection. These participants will be removed from CCMDD and will need to return to $\mathrm{CDC}$ for ART collection and adherence counselling where necessary.

\section{Data collection and management}

Clinical data including ART regimen changes, entry into CCMDD, new clinical diagnoses and receipt of intensive adherence counselling will be collected during study clinic visits or clinical chart review. Data will be captured by study staff using laptops and standardised electronic case report forms in the iDataFax system (DF/Net Research, Seattle, USA). All data entry will undergo three stages of quality control including immediate source document review, internal quality audits and weekly quality reports generated by iDataFax.

\section{Statistical methods}

A descriptive analysis of baseline sociodemographic variables will be used to assess comparability of the two study

\begin{tabular}{llc}
\hline Table 2 Sample size and power estimate & & \\
\hline $\begin{array}{l}\text { Non-inferiority design hypothesis, assuming standard of care arm } \\
\text { achieves } 80 \%\end{array}$ & Power (beta) & $\begin{array}{l}\text { Estimated total sample } \\
\text { size }\end{array}$ \\
\hline Rule out $75 \%$ against true intervention $85 \%$ & $80 \%$ & 158 \\
Rule out $75 \%$ against true intervention $85 \%$ & $90 \%$ & 220 \\
Rule out $70 \%$ against true intervention $80 \%$ & $80 \%$ & 396 \\
Rule out $70 \%$ against true intervention $80 \%$ & $90 \%$ & 550 \\
Rule out $72.5 \%$ against true intervention $80 \%$ & $80 \%$ & 704 \\
\hline
\end{tabular}

Bold values correspond with the selected target sample size. 
arms. The primary analysis will estimate the absolute difference in proportions achieving the primary outcome between the intervention and control arms, assessed using the $\chi^{2}$ test. For binary secondary outcomes, the absolute difference in proportions will be assessed using the $\chi^{2}$ test. For continuous secondary outcomes, means in each arm will be compared using the unpaired Student's t-test. We will perform bivariate and multivariable logistic regression to identify independent risk factors for reaching the primary outcome. Each covariate will be assessed for statistical significance in a bivariate logistic regression. Potential risk factors that have been identified in the literature $\left(\mathrm{eg}\right.$, age, sex ${ }^{51}$ ) will also be assessed as covariates in a multivariable regression. Data will be analysed using SAS and R (R Foundation for Statistical Computing).

\section{Secondary costing analysis}

An activity-based micro-costing approach will be used to estimate the cost per patient and per patient virologically suppressed and retained in care in the intervention arm compared with the standard of care arm. Time and motion studies, observing multiple staff members over several visits, will allow an estimation of the average time taken to complete each step of the clinic visit (VL testing, clinical assessment, counselling) for both study arms. Time spent on research purposes (eg, data collection) will be noted separately from time spent on clinical care. Standardised activity-based cost menus will be used to collect site costs, including start-up costs, human resources, supplies, VL test costs and other expenses. When data are not available from our cohort, we will use data from population-based South African studies. Additional cost data may be obtained from health facilities, published government information on labour costs and health economics literature. Analyses will follow the guidelines for costing HIV interventions ${ }^{52}$ and will reflect the provider perspective. Costs will be categorised as fixed or variable. Variable costs indicate which costs could change (eg, less expensive POC HIV VL assay) and influence the estimates from the study. We will also collect data on costs incurred by participants, to determine whether POC VL testing is cost-effective from a patient and societal perspective. The micro-costing data, time and motion studies and clinical outcomes will be used to estimate the average cost per HIV-positive patient achieving viral suppression and retained in care in the intervention arm compared with standard of care. Furthermore, these data will allow process evaluation of the mechanism by which the different components of the intervention (POC testing and enrolled nurses) contribute to the achievement of study outcomes, including costs saved and incurred.

\section{ETHICS, MONITORING AND DISSEMINATION}

The trial has been approved by the University of KwaZulu-Natal Biomedical Research Ethics Committee (BFC296/16) and the University of Washington Institutional Review Board (STUDY00001466). Permission to conduct the study in the PCZ CDC was provided by the eThekwini Municipality Health Unit. Eligible patients will be asked to provide written informed consent for study procedures and sample storage. All participants will receive a small financial compensation (ZAR 100, approximately US\$7.5) for their time, transport and inconvenience after each study visit in accordance with South African Research Council Guidelines. ${ }^{53}$ A data monitoring committee is not required as the risk of significant harms from study procedures is low. Any breaches in confidentiality, study protocol or adverse events attributable to this study will be reported to the above institutional review boards. Results will be presented at national and international conferences and published in academic, peer-reviewed journals.

\section{Trial status}

The trial was registered on clinicaltrials.gov (NTC03066128) on 22 February 2017. Enrolment started on 24 February 2017 and is predicted to be completed in August 2017.

\section{DISCUSSION}

New models of HIV care are urgently required if the UNAIDS 90-90-90 targets are to be reached. To our knowledge, this is the first randomised controlled trial investigating a combined intervention of POC HIV VL testing and task shifting. If clinically effective and affordable, this strategy may contribute to the successful scale up of $\mathrm{VL}$ monitoring and ART coverage. The STREAM study will also generate much needed real-world data to improve mathematical models of VL testing strategies. ${ }^{1825}$

Results from this study of the Xpert HIV-1 VL may be applicable to other quantitative POC VL assays on the market with similar performance characteristics. The SAMBA I/II semi-Q (Diagnostics for the Real World, Cambridge, United Kingdom) ${ }^{54}$ Liat (Roche Diagnostics, Basel, Switzerland) ${ }^{55}$ and Alere Q NAT (Alere, Waltham, Massachusetts, USA) ${ }^{56}$ have all been evaluated in Southern Africa, each with strengths and limitations. The Xpert HIV-1 VL is limited by the need for centrifuging blood samples to obtain plasma and a 90 min processing time. While Cepheid is working to address both of these issues, the widespread use of the GeneXpert platform for TB diagnostics means that the Xpert HIV-1 VL is particularly amenable to rapid scale up in several low-income and middle-income countries. ${ }^{57}$

The STREAM study design incorporates two forms of differentiated care: task shifting to enrolled nurses in the intervention arm and community ART collection for stable participants in both arms. Task shifting ART provision from physician-led secondary care services to nurses in primary care facilities has been successfully implemented in Southern Africa. ${ }^{58}{ }^{59}$ We hypothesise that further task shifting from professional to enrolled nurses may be a cost-effective and gentler 'step down' prior to community ART collection and that POC VL testing will 
allow more effective triage into community ART collection programme. ${ }^{14161738}$ Removing the need to reattend for VL results could decrease clinic visits within these programme by $33 \%-50 \%$.

A limitation of our clinical implementation trial is the relatively small sample size and conducting the study at a single site. The diverse population and urban setting of the trial may mean that results are not generalisable across all parts of Southern Africa. Nevertheless, findings from STREAM may be used to design POC VL studies in other priority populations and settings, including those with high incidence of virological failure (eg, adolescents) and rural areas with limited laboratory access. A second limitation may be that financial compensation provided in the study could increase retention in care compared with non-study settings. However, the reimbursements are relatively small and will be offered to all participants, so are unlikely to impact on comparisons of the primary outcome between arms.

Achieving the 90-90-90 targets requires a large and rapid scale up of HIV VL monitoring, as well as strategies that make HIV programme more efficient and facilitate retention in care. The STREAM study will provide evidence as to whether POC VL testing within a differentiated care model can help achieve these goals.

\section{Author affiliations}

${ }^{1}$ Centre for the AIDS Programme of Research in South Africa (CAPRISA), University of KwaZulu-Natal, Durban, KwaZulu-Natal, South Africa

${ }^{2}$ Discipline of Public Health Medicine, School of Nursing and Public Health, University of KwaZulu-Natal, Durban, KwaZulu-Natal, South Africa

${ }^{3}$ Department of Global Health, Schools of Medicine and Public Health, University of Washington, Seattle, Washington, USA

${ }^{4}$ Prince Cyril Zulu Communicable Disease Clinic, Durban Municipality, Durban, KwaZulu-Natal, South Africa

${ }^{5}$ Department of Virology, Inkosi Albert Luthuli Central Hospital, Durban, KwaZuluNatal, South Africa

${ }^{6}$ School of Laboratory Medicine and Medical Sciences, University of KwaZulu-Natal, Durban, KwaZulu-Natal, South Africa

${ }^{7}$ National Health Laboratory Service, Durban, KwaZulu-Natal, South Africa

${ }^{8}$ Department of Medicine, School of Medicine, University of Washington, Seattle, Washington, USA

${ }^{9}$ Department of Epidemiology, School of Public Health, University of Washington, Seattle, USA

${ }^{10}$ MRC-CAPRISA HIV-TB Pathogenesis and Treatment Research Unit, Doris Duke Medical Research Institute, University of KwaZulu-Natal, Durban, KwaZulu-Natal, South Africa

${ }^{11}$ Department of Epidemiology, Columbia University, New York City, USA

Acknowledgements The authors would like to thank all participants in the study and acknowledge the work and support of staff at the Centre for the AIDS Programme of Research in South Africa, the University of Washington, Ethekwini Municipality and the Prince Cyril Zulu Communicable Diseases Clinic.

Contributors PD and NG conceived the trial and are the co-principal investigators. PD, NG, JD, JQA, NS, HN, NN, PM, KM, TS, KN, SAK and CC designed the study. PD, NG and JD wrote the study protocol. DD performed sample size calculations and the statistical analysis strategy. RB devised the secondary costing analysis. All authors contributed to the manuscript and consented to final publication.

Funding STREAM is funded by the US National Institute for Health (NIH) (Al124719$01)$ and the University of Washington's Center for AIDS Research. Cepheid Inc. loaned the GeneXpert instruments for this study at no cost. NIH and Cepheid have no role in study design, manuscript submission or collection, management, analysis or interpretation of study data.
Competing interests None declared.

Ethics approval University of KwaZulu-Natal Biomedical Research Ethics Committee (BFC296/16) and the University of Washington Institutional Review Board (STUDY00001466).

Provenance and peer review Not commissioned; externally peer reviewed.

Open Access This is an Open Access article distributed in accordance with the Creative Commons Attribution Non Commercial (CC BY-NC 4.0) license, which permits others to distribute, remix, adapt, build upon this work non-commercially, and license their derivative works on different terms, provided the original work is properly cited and the use is non-commercial. See: http://creativecommons.org/ licenses/by-nc/4.0/

(c) Article author(s) (or their employer(s) unless otherwise stated in the text of the article) 2017. All rights reserved. No commercial use is permitted unless otherwise expressly granted.

\section{REFERENCES}

1. Cohen MS, Chen YQ, McCauley M, et al. Prevention of HIV1 infection with early antiretroviral therapy. $N$ Engl J Med 2011;365:493-505.

2. Lundgren JD, Babiker AG, Gordin F, et al. Initiation of antiretroviral therapy in early asymptomatic HIV infection. $N$ Engl J Med 2015;373:795-807.

3. Danel C, Moh R, Gabillard D, et al. A trial of early antiretrovirals and isoniazid preventive therapy in Africa. $N$ Engl $\mathrm{J}$ Med 2015;373:808-22.

4. World Health Organisation. Consolidated guidelines on the use of antiretroviral drugs for treating and preventing HIV infection: recommendations for a public health approach. 2nd ed : Geneva, Switzerland, 2016.

5. Joint United Nations Programme on HIV/AIDS. 90-90-90 An ambitious treatment target to help end the AIDS epidemic. Geneva, Switzerland, 2014.

6. Joint United Nations Programme on HIV and AIDS. Fact sheet November 2016. Geneva, Switzerland, 2016.

7. Hontelez JA, Chang AY, Ogbuoji O, et al. Changing HIV treatment eligibility under health system constraints in sub-Saharan Africa: investment needs, population health gains, and cost-effectiveness. AIDS 2016:30:2341-50.

8. Govindasamy D, Ford N, Kranzer K. Risk factors, barriers and facilitators for linkage to antiretroviral therapy care: a systematic review. AIDS 2012;26:2059-67.

9. Fox MP, Rosen S. A new cascade of HIV care for the era of "treat all". PLoS Med 2017; 14:e1002268.

10. Duncombe C, Rosenblum S, Hellmann N, et al. Reframing HIV care: putting people at the centre of antiretroviral delivery. Trop Med Int Health 2015;20:430-47.

11. Holmes CB, Sanne I. Changing models of care to improve progression through the HIV treatment cascade in different populations. Curr Opin HIV AIDS 2015;10:447-50.

12. Babigumira JB, Castelnuovo $B$, Lamorde $M$, et al. Potential impact of task-shifting on costs of antiretroviral therapy and physician supply in Uganda. BMC Health Serv Res 2009;9:192.

13. Cawley C, Nicholal S, Szumilin E, et al, 2016. Six-monthly appointments as a strategy for stable antiretroviral therapy patients: evidence of its effectiveness from seven years of experience in a Medecins Sans Frontieres supported programme in Chiradzulu district, Malawi. In: 21st International AIDS Conference, Durban

14. Decroo T, Koole O, Remartinez D, et al. Four-year retention and risk factors for attrition among members of community ART groups in Tete, Mozambique. Trop Med Int Health 2014;19:514-21.

15. Okoboi S, Ding E, Persuad S, et al. Community-based ART distribution system can effectively facilitate long-term program retention and low-rates of death and virologic failure in rural Uganda. AIDS Res Ther 2015;12:37.

16. Wilkinson L, Harley B, Sharp J, et al. Expansion of the adherence club model for stable antiretroviral therapy patients in the Cape Metro, South Africa 2011-2015. Trop Med Int Health 2016;21:743-9.

17. Vogt F, Kalenga L, Lukela J, et al. Brief report: decentralizing ART supply for stable HIV patients to community-based distribution centers: program outcomes from an urban context in Kinshasa, DRC J Acquir Immune Defic Syndr 2017;74:326-31.

18. Phillips A, Shroufi A, Vojnov L, et al. Sustainable HIV treatment in Africa through viral-load-informed differentiated care. Nature 2015;528:S68-S76. 
19. Lecher S, Ellenberger D, Kim AA, et al. Scale-up of HIV viral load monitoring-seven Sub-Saharan African countries. MMWR Morb Mortal Wkly Rep 2015;64:1287-90.

20. Peter T, Ellenberger D, Kim AA, et al. Early antiretroviral therapy initiation: access and equity of viral load testing for HIV treatment monitoring. Lancet Infect Dis 2017;17:2014-7.

21. World Health Organization. HIV diagnostic tests in low and middleincome countries: forecasts of global demand for 2014. Geneva, 20182015.

22. Usdin M, Guillerm M, Calmy A, Needs P. Patient needs and point-ofcare requirements for HIV load testing in resource-limited settings. $J$ Infect Dis 2010;201 Suppl 1:S73-7.

23. Shafiee H, Wang S, Inci F, et al. Emerging technologies for point-ofcare management of HIV infection. Annu Rev Med 2015;66:387-405.

24. Stevens W, Gous N, Ford N, et al. Feasibility of HIV point-of-care tests for resource-limited settings: challenges and solutions. BMC Med 2014;12:173.

25. Phillips AN, Cambiano V, Nakagawa F, et al. Point-of-care viral load testing for Sub-Saharan Africa: informing a target product profile. Open Forum Infect Dis 2016;3:ofw161.

26. Estill J, Egger M, Blaser N, et al. Cost-effectiveness of point-of-care viral load monitoring of antiretroviral therapy in resource-limited settings: mathematical modelling study. AIDS 2013;27:1483-92.

27. UNITAID. HIVIAIDS diagnostic technology landscape. 5th ed. Geneva, 2015

28. Drain PK, Garrett NJ. The arrival of a true point-of-care molecular assay-ready for global implementation? Lancet Glob Health 2015;3:e663-e664.

29. Garrett NJ, Drain PK, Werner L, et al. Diagnostic accuracy of the point-of-care expert HIV-1 viral load assay in a South African HIV clinic. J Acquir Immune Defic Syndr 2016;72:e45-e48.

30. Ceffa S, Luhanga R, Andreotti M, et al. Comparison of the cepheid geneXpert and abbott M2000 HIV-1 real time molecular assays for monitoring HIV-1 viral load and detecting HIV-1 infection. J Virol Methods 2016;229:35-9.

31. Moyo S, Mohammed T, Wirth KE, et al. Point-of-care cepheid Xpert HIV-1 viral load test in rural African communities is feasible and reliable. J Clin Microbiol 2016;54:3050-5.

32. Gous N, Scott L, Berrie L, et al. Options to expand HIV viral load testing in South Africa: evaluation of the GeneXpert@ HIV-1 viral load assay. PLoS One 2016;11:e0168244.

33. Munjanja O, Kibuka S, Dovlo D. The nursing workforce in subSaharan Africa. Geneva, Switzerland: International Council of Nurses, 2005.

34. WHO Africa Health Workforce Observatory. Definitions of the 23 health workforce categories [Internet], 2007. http://apps.who.int/ globalatlas/docs/HRH_HWO/HTML/Dftn.htm. (accessed 15 Mar 2017)

35. South African National Department of Health. National consolidated guidelines for the prevention of mother to child transmission of HIV (PMTCT) and the management of HIV in children, adolescents and adults. Pretoria, South Africa, 2015.

36. Shisana O, Rehle T, Simbayi LC, et al. South African national HIV prevalence, incidence and behaviour survey, 2012: HSRC Press, 2014.

37. South African National Department of Health. Implementation of the universal test and treat strategy for HIV positive patients and differentiated care for stable patients. Pretoria, South Africa, 2016.

38. Health Systems Trust. Central chronic medicine dispensing and distribution programme (CCMDD), 2015. http://www.hst.org.za/ projects/central-chronic-medicine-dispensing-and-distributionprogramme-ccmdd (accessed 6 Mar 2017).

39. The South African National Department of Health. Standard treatment guidelines and essential medicines list for South Africa: primary health care level. Pretoria, South Africa, 2014.

40. Peltzer K, Simbayi L, Kalichman S, et al. Alcohol use in three different inner cities in South Africa: AUDIT-C and CAGE. J Psychol Africa 2007;17:99-104.
41. Seth $P$, Glenshaw M, Sabatier JH, et al. AUDIT, AUDIT-C, and AUDIT-3: drinking patterns and screening for harmful, hazardous and dependent drinking in Katutura, Namibia. PLoS One 2015;10:e0120850.

42. Bernstein M, Phillips T, Zerbe A, et al. Intimate partner violence experienced by HIV-infected pregnant women in South Africa: a cross-sectional study. BMJ Open 2016;6:e011999.

43. Jewkes R, Gibbs A, Jama-Shai N, et al. Stepping stones and creating futures intervention: shortened interrupted time series evaluation of a behavioural and structural health promotion and violence prevention intervention for young people in informal settlements in Durban, South Africa. BMC Public Health 2014;14:1325.

44. Bhana A, Rathod SD, Selohilwe O, et al. The validity of the patient health questionnaire for screening depression in chronic care patients in primary health care in South Africa. BMC Psychiatry 2015;15:118.

45. Baron EC, Davies T, Lund C. Validation of the 10-item centre for epidemiological studies depression scale (CES-D-10) in Zulu, Xhosa and Afrikaans populations in South Africa. BMC Psychiatry 2017;17:6.

46. Kosack CS, de Kieviet W, Bayrak K, et al. Evaluation of the Nova statsensor $\AA$ Xpress $(T M)$ creatinine point-of-care handheld analyzer. PLoS One 2015;10:e0122433.

47. Minnings K, Kerns E, Fiore M, et al. Chronic kidney disease prevalence in Rivas, Nicaragua: use of a field device for creatinine measurement. Clin Biochem 2015:48:456-8.

48. van Lint CL, van der Boog PJ, Romijn FP, et al. Application of a point of care creatinine device for trend monitoring in kidney transplant patients: fit for purpose? Clin Chem Lab Med 2015;53:1547-56.

49. Gous NM, Scott LE, Potgieter J, et al. Implementation and operational research: implementation of multiple point-of-care testing in 2 HIV antiretroviral treatment clinics in South Africa. J Acquir Immune Defic Syndr 2016;71:e34-43.

50. Gous N, Scott L, Potgieter J, et al. Feasibility of performing multiple point of care testing for HIV anti-retroviral treatment initiation and monitoring from multiple or single fingersticks. PLoS One 2013;8:e85265-10.

51. Grobler A, Cawood C, Khanyile D, et al. Progress of UNAIDS 90-9090 targets in a district in KwaZulu-Natal, South Africa, with high HIV burden, in the HIPSS study: a household-based complex multilevel community survey. Lancet HIV 2017;3018:1-9.

52. Joint United Nations Programme on HIV/AIDS. Costing guidelines for HIV prevention strategies. Geneva, Switzerland, 2000.

53. National Health Research Ethics Council (NHREC). Payment of trial participants in South Africa: ethical considerations for research ethics committees: South African Department of Health, 2012.

54. Ritchie AV, Ushiro-Lumb I, Edemaga D, et al. SAMBA

HIV semiquantitative test, a new point-of-care viral-loadmonitoring assay for resource-limited settings. $J$ Clin Microbiol 2014:52:3377-83.

55. Scott L, Gous N, Carmona S, et al. Laboratory evaluation of the Liat HIV Quant (IQuum) whole-blood and plasma HIV-1 viral load assays for point-of-care testing in South Africa. J Clin Microbio 2015;53:1616-21.

56. Jani IV, Meggi B, Vubil A, et al. Evaluation of the whole-blood Alere Q NAT point-of-care RNA assay for HIV-1 viral load monitoring in a primary health care setting in Mozambique. $J$ Clin Microbiol 2016;54:2104-8.

57. UNITAID. Largest ever roll-out of GeneXpert $\circledast$ rapid TB test machines. 2013 http://www.unitaid.org/en/press-releases/1266largest-ever-roll-out-of-genexpert-rapid-tb-test-machines (accessed 4 Apr 2017).

58. Kredo T, Ford N, Adeniyi FB, et al. Decentralising HIV treatment in lower- and middle-income countries. Cochrane Database Syst Rev 2013:CD009987.

59. Kredo T, Adeniyi FB, Bateganya M, et al. Task shifting from doctors to non-doctors for initiation and maintenance of antiretroviral therapy. Cochrane Database Syst Rev 2014:CD007331. 